\title{
Influence of Different Tougheners on the Properties of PC/PBT Alloy
}

\author{
Pengwei Shi, Junjie Tang, Hao Duan* \\ Fine-Blend Polymer (Shanghai) Co., LTD., Shanghai, China \\ Email: ^duanhao06@hotmail.com
}

How to cite this paper: Shi, P.W., Tang, J.J. and Duan, H. (2021) Influence of Different Tougheners on the Properties of PC/PBT Alloy. Journal of Materials Science and Chemical Engineering, 9, 70-82. https://doi.org/10.4236/msce.2021.911005

Received: October 21, 2021

Accepted: November 27, 2021

Published: November 30, 2021

Copyright $\odot 2021$ by author(s) and Scientific Research Publishing Inc. This work is licensed under the Creative Commons Attribution International License (CC BY 4.0).

http://creativecommons.org/licenses/by/4.0/ (c) (i) Open Access

\begin{abstract}
This paper focuses on the influence of glycidyl methacrylate functionalized polyolefin elastomer (SOG-03) on the properties of PC/PBT alloys, and also made a contrastive analysis with ethylene-methyl acrylate-glycidyl methacrylate terpolymer (EMA-co-GMA) and methyl methacrylate-butadiene-styrene terpolymer (MBS), the common toughener in $\mathrm{PC} / \mathrm{PBT}$ alloy. The impact performance test results of PC/PBT alloys with different addition of SOG-03 showed that the brittle-ductile transition began when SOG-03 content reached $3 \mathrm{wt} \%$. The microstructure, differential scanning calorimeter (DSC) and multi-extrusion process results of PC/PBT alloys all showed that SOG-03 tends to be dispersed in PBT phase and the dispersed SOG-03 presents typical rubber-toughened polymer morphology. The toughening efficiency of MBS on PC/PBT alloy was much lower than EMA-co-GMA and SOG-03, and showed a worse processing stability after multi-extrusion process and long-term thermal ageing properties. The EMA-co-GMA and SOG-03 toughened PC/PBT alloys showed an equivalent toughness, while the PC/PBT alloy with SOG-03 showed a better processing stability during the multi-extrusion process and long-term thermal ageing property when the thermal aging time is more than $600 \mathrm{~h}$.
\end{abstract}

\section{Keywords}

PC/PBT Alloy, Toughener, Microstructure, Thermal Stability, Processing Stability

\section{Introduction}

Polycarbonate (PC) is a kind of typical amorphous polymer with good mechanical property, heat resistance and electrical property, especially outstanding impact performance, which ranks first in engineering plastics. However, there exist 
a large number of benzene rings in the molecular chains of PC. The molecular rigidity and steric hindrance of $\mathrm{PC}$ are large, contributing to high melting temperature of the polymer, high viscosity of the melt, difficult processing, large residual stress of the product and stress cracking tendency [1] [2] [3]. Therefore, it's necessary to modify its properties to meet practical applications. PBT is a kind of thermoplastic polymer with excellent chemical resistance and flowability. However, its lower notched impact strength and heat distortion temperature under higher load become obstacle to further applications. Thence, complementary advantages could be achieved if PC blended with PBT, which can overcome the shorting comings of PC such as poor chemical resistance and difficulty in molding, and make up for the defects of PBT such as poor heat resistance and low impact strength [2] [3] [4]. However, the blending of PC and PBT is a typical blending system of crystalline and non-crystalline polymer, which has poor adhesion of phase interface. Simple melt blending will cause phase separation that contributes to poor impact toughness and limits the application of PC/PBT alloy.

However, choosing proper toughening compatibilizer is crucial for preparing PC/PBT alloy with good property. Among them, ethylene-methyl acrylate-glycidyl methacrylate terpolymer (EMA-co-GMA), ethylene-butyl acrylate-glycidyl methacrylate terpolymer (EBA-co-GMA) and methyl methacrylate-butadiene-styrene terpolymer (MBS) with core-shell structure have been proven to be effective in toughening compatibilizers in PC/PBT alloy [5] [6] [7] [8] [9]. Recently, polyolefin elastomer modified with glycidyl methacrylate has developed into new varieties of toughening agent for polyester due to good toughening efficiency, heat resistance and easy accessibility [4] [10] [11].

Herein, a new type of POE-g-GMA product (SOG-03) was employed as toughening compatibilizers in $\mathrm{PC} / \mathrm{PBT}$ alloy, which improves the shortcomings of large odor and high residual monomer content in the production of traditional POE-g-GMA by the reactive extrusion process. Meanwhile, EMA-co-GMA and MBS, are usually used as toughener in PC/PBT alloy, as a comparison (the ratio of $\mathrm{PC} / \mathrm{PBT}$ in this work is $70 / 30$ ). The transesterification in the process of blending $\mathrm{PC} / \mathrm{PBT}$ alloy is a significant factor for deteriorating properties of composite material. Li et al. [12] [13] demonstrated that anhydrous sodium dihydrogen phosphate has the best effect on the inhibition of ester exchange of alloy materials, and the comprehensive properties are optimal when the addition amount is $0.3 \mathrm{wt} \%$. Therefore, the PC/PBT alloys prepared in this work were added with $0.3 \mathrm{wt} \%$ anhydrous sodium dihydrogen phosphate as a transesterification inhibitor.

\section{Experimental Section}

\subsection{Materials}

Polycarbonate: 1100, medium viscosity slice, Lotte Chemical Co., PBT: 6100, medium viscosity slice, Xinjiang Lanshan Tunhe Polyester Co., Ltd., SOG-03: POE-g-GMA, GMA content (2 - 3 wt\%), Fine-blend Polymer (Shanghai) Co., 
Ltd., EMA-co-GMA: ethylene-methyl acrylate-glycidyl methacrylate terpolymer, GMA content (8 wt\%), commercially available; MBS: M-711, core-shell structured methyl methacrylate-butadiene-styrene terpolymer, Japan Kaneka Chemical Industry Co., Ltd.; anhydrous sodium dihydrogen phosphate: $\mathrm{NaH}_{2} \mathrm{PO}_{4}$, Tianjin Guangfu Fine Chemical Co., Ltd.; antioxidant: 1010/168, Jilin Petrochemical Company Synthetic Resin Factory.

\subsection{Characterization}

Twin-screw extruder: KS-36, Kunshan Kexin Rubber \& Plastic Machinery Co., Ltd.; Injection Molding Machine: JN88E, Zhenxiong Machinery Co., Ltd.; Electronic Universal Testing Machine: CMT6104, Shenzhen Xinsansi Material Testing Co., Ltd.; Impact Testing Machine: XC-2.75, Hebei Chengde Precision Testing Machine Co., Ltd.; Melt Flow Rate Instrument (MFR): ZRZ1452, Shenzhen Xinsisi Material Testing Co., Ltd.; Differential Scanning Calorimeter (DSC): DSC200F3, NETZSCH, Germany; Color Difference Meter: Color Eye 7000A Benchtop Spectrophotometer, X-rite Corporation, USA; Scanning Electron Microscopy (SEM): JSM-6360LV, JEOL Ltd; Ventilation Aging Test Chamber: HOC-HQ45A, Shanghai Hengyi Electronic Technology Co., Ltd.

\subsection{Preparation of PC/PBT Alloys}

1) Conventional extrusion process: The dried PC, PBT (PC $120^{\circ} \mathrm{C} / 4-6 \mathrm{~h}$, $\mathrm{PBT} \sim 80^{\circ} \mathrm{C} / 4-6 \mathrm{~h}$ ) and toughener were mixed with other components in a certain ratio (the ratio of $\mathrm{PC} / \mathrm{PBT}$ was $70 / 30$ in this work), and then extruded and granulated; the temperature of the twin-screw extruder was set at $220^{\circ} \mathrm{C}-250^{\circ} \mathrm{C}$, and the rotational speed of the main engine was $300 \mathrm{r} / \mathrm{min}$. The samples obtained after extrusion granulation were dried at $105^{\circ} \mathrm{C}$ for $4 \mathrm{~h}$, and then injection molded into various standard splines by an injection molding machine, and then subjected to different performance tests.

2) Distributed extrusion process: Firstly, according to the conventional extrusion, the reference sample was obtained, where SOG-03 was used as the toughener; the dried PC and SOG-03 were extruded and granulated in a certain ratio, and after drying the particles, the dried PBT and other auxiliary agents were extruded again to produce granules, and distributed extrusion sample $1 \#$ of multi-extrusion process could be obtained after being dried, other processes were consistent with conventional extrusion experiments; dried PBT and SOG-03 were extruded and granulated at a certain ratio, dried PC and other auxiliary agents were again extruded and granulated after drying the particles, distributed extrusion sample $2 \#$ were obtained, and other processes were consistent with conventional extrusion experiments.

3) Multi-extrusion process: The dried PC, PBT and toughening agent were uniformly mixed in a certain ratio and then extruded and granulated to obtain a reference sample; the reference samples were dried at $105^{\circ} \mathrm{C}$ for 4 hours and then granulated by an extruder to obtain multiple sample $1 \#$ of multi-extrusion process, sample 2, sample 4, sample 6 and sample 8 can be obtained according to 
the different extrusion times by analogy. After the samples were dried, they were molded into standard samples by injection molding machine, and then tested the performance.

\subsection{Performance Test}

Tensile property was tested according to ISO 527 at a tensile rate of $50 \mathrm{~mm} / \mathrm{min}$, bending property was tested according to ISO 178 with a pressure head speed of $2 \mathrm{~mm} / \mathrm{min}$, notched impact property was tested according to ISO 179 with a pendulum energy of $4 \mathrm{~J}$. Melt flow rate (MFR) was tested in accordance with ASTM 1133 at a test temperature of $250^{\circ} \mathrm{C}$ and a load of $5 \mathrm{~kg}$. Differential scanning calorimetry (DSC) was performed under $\mathrm{N}_{2}$. The temperature was increased from room temperature to $300^{\circ} \mathrm{C}$ for the first time at a rate of $20^{\circ} \mathrm{C} / \mathrm{min}$, and the temperature was kept for $5 \mathrm{~min}$ to eliminate the thermal history of the sample, then samples were cyclic scanned at a drop/rise temperature rate of $10^{\circ} \mathrm{C} / \mathrm{min}$. The value of color difference (the value of $\Delta \mathrm{E}$ ): The particles obtained by conventional extrusion and multi-extrusion process were respectively molded into a standard color plate by an injection molding machine, and then the value of $\Delta \mathrm{E}$ was tested. Scanning electron microscope (SEM): Representative impact section was selected and the microstructure was observed by SEM after gold spraying of $40 \mathrm{~s}$. Long-term thermal aging property: The injection molding spline was suspended in the ventilation aging box, the thermal aging temperature was set to $150^{\circ} \mathrm{C}$, the sampling time were $200 \mathrm{~h}, 400 \mathrm{~h}, 600 \mathrm{~h}$ and $800 \mathrm{~h}$ respectively, and then the property testing was performed.

\section{Results and Discussion}

\subsection{The Influence of SOG-03 Content on the Notched Impact Strength of PC/PBT Alloy}

The blend of PC and PBT is a typical amorphous-crystalline polymer blending system, types and content of compatible tougheners are crucial for properties and processability of blending alloys. The notched impact strength of PC/PBT alloy with different content of SOG-03 is shown in Figure 1. The impact strength of $\mathrm{PC} / \mathrm{PBT}$ alloy without toughener is $6.6 \mathrm{KJ} / \mathrm{m}^{2}$, which is typical brittle material, and its morphology will be mentioned later. With the increased addition of SOG-03, the notched impact strength of PC/PBT alloy showed an increasing trend clearly. When the content was up to $3 \mathrm{wt} \%$, notched impact strength would mutate that showed a distinct brittle-ductile transition. Then notched impact strength slightly increased with the increased content of SOG-03 when the alloy turned into super toughness, which explained excessive addition of the toughener had a limited effect on further improving the impact strength and would even cause a decrease in the mechanical properties of the alloy.

\subsection{The Influence of SOG-03 Content on the Melt and Crystallization Behavior of PC/PBT Alloy}

The DSC curves of PC/PBT alloy were also influenced by the content of compatible 


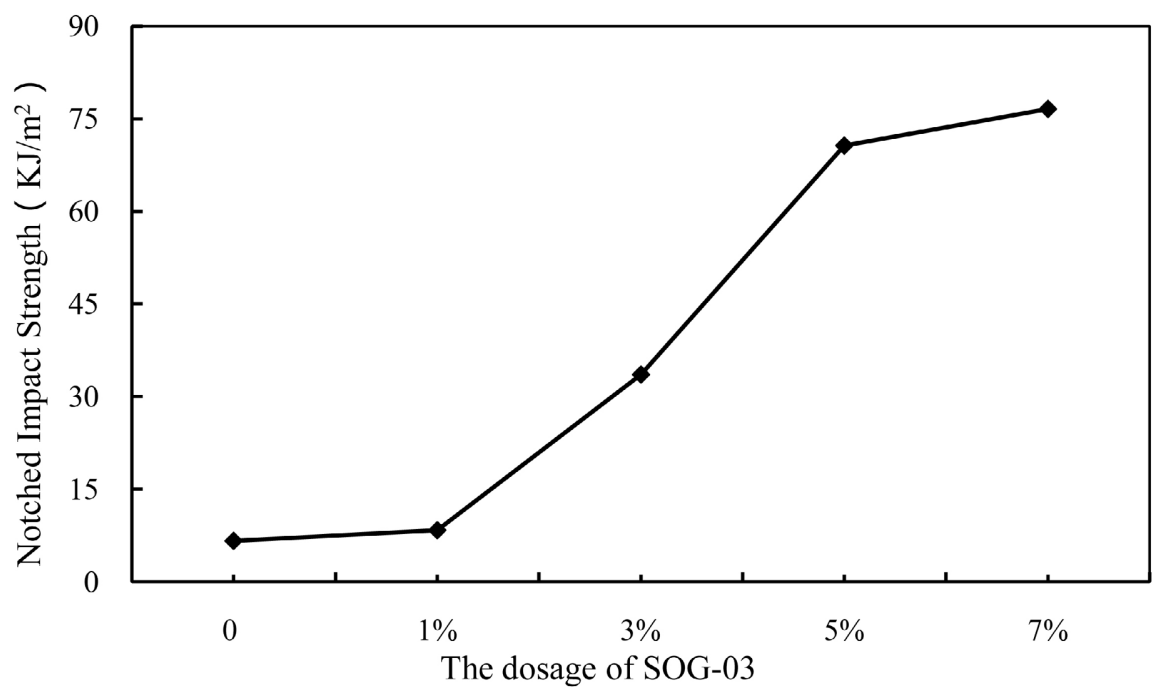

Figure 1. The influence of SOG-03 content (wt\%) on the notched impact strength of $\mathrm{PC} / \mathrm{PBT}$ alloy.

tougheners; Figure 2(A) and Figure 2(B) shows the melting and crystallization behavior of PC/PBT alloy with different SOG-03, and the DSC data were listed in Table 1. The $\mathrm{T}_{\mathrm{g}}$ of PC phase drifted toward low temperature that proved compatibility of two-phase system could be improved with the increase of content of reactive compatibilizer, SOG-03. What's more, $\mathrm{T}_{\mathrm{m}}, \mathrm{T}_{\mathrm{c}}, \Delta \mathrm{H}_{\mathrm{m}}$ and $\Delta \mathrm{H}_{\mathrm{c}}$ of $\mathrm{PBT}$ phase in $\mathrm{PC} / \mathrm{PBT}$ alloy showed a transparent downward trend with the increase of SOG-03 content. SOG-03 was mainly dispersed in PBT phase with the increase of SOG-03 content, contributing to the reduction of the PBT segment activity and affecting the crystallization and fusion of the PBT phase. In order to verify whether SOG-03 tended to be dispersed in the PBT phase in PC/PBT alloy, the microstructure of $\mathrm{PC} / \mathrm{PBT}$ alloy was observed and multi-extrusion process was conducted.

\subsection{The Influence of Toughener Type on the Microstructure of PC/PBT Alloy}

Figure 3 shows the microstructure of impact section at different magnification, in which PC/PBT alloys were added with different tougheners. It can be clearly observed that at different magnification, the impact section of PC/PBT alloy without tougheners showed typical brittle fracture (Figure 3(A) \& Figure 3(a)) while the other three showed typical ductile fracture if $6 \mathrm{wt} \%$ EMA-co-GMA (Figure 3(B)), SOG-03 (Figure 3(C)) and MBS (Figure 3(D)) were added into PC/PBT alloys respectively, which indicated these three types of tougheners could all make the impact property of the alloy better.

The impact fracture surface of $\mathrm{PC} / \mathrm{PBT}$ alloys with different tougheners under different magnification were added (as shown in Figures 3(b)-(d)), the results showed the enlarged impact section (Figure 3(b)) of PC/PBT alloy added with EMA-co-GMA was rough while that added with MBS was very smooth (Figure 3(d)), and neither impact section could help judge dispersion of tougheners in 


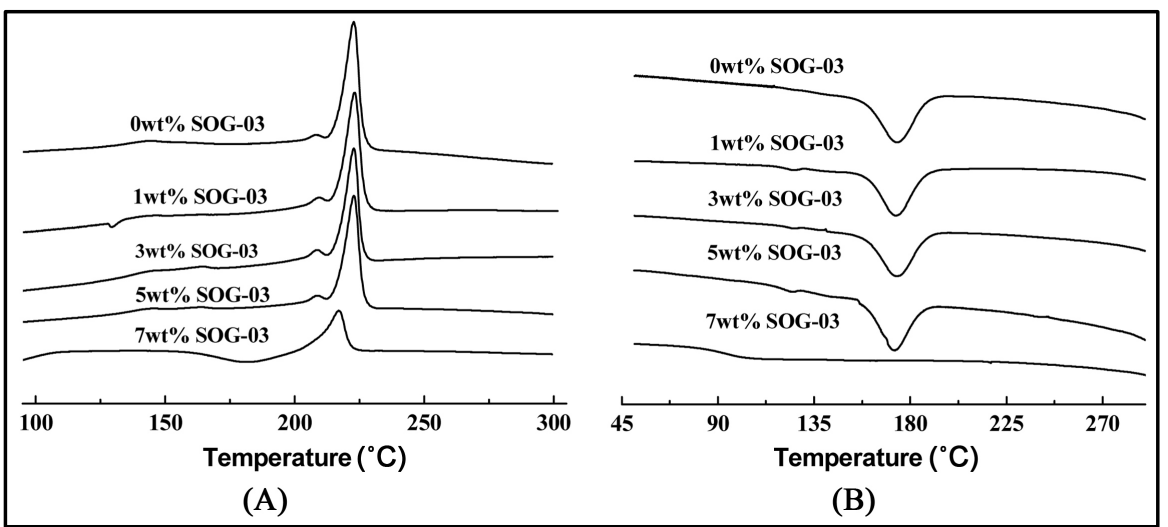

Figure 2. The melting (A) and crystallization (B) curves of PC/PBT alloy with different content of SOG-03.
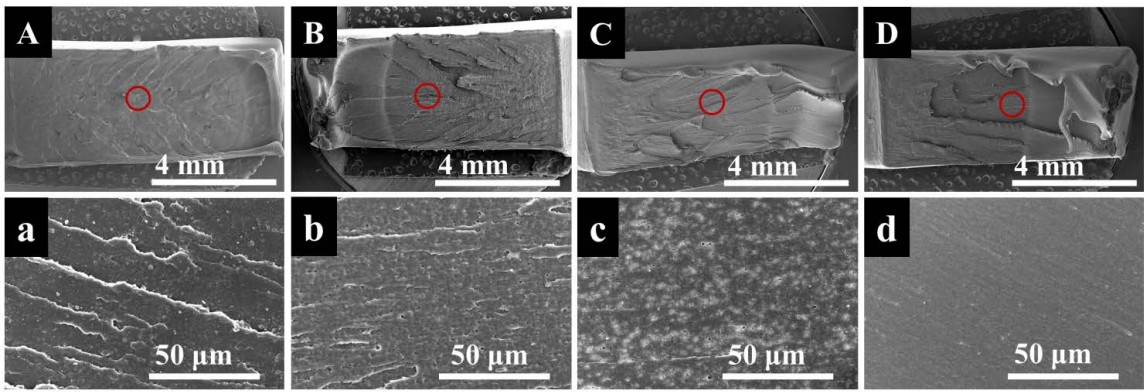

Figure 3. The microstructure of PC/PBT alloy with different toughener ((A) (a) Without toughener; (B) (b) $6 \mathrm{wt} \%$ EMA-co-GMA; (C) (c) $6 \mathrm{wt} \%$ SOG-03; (D) (d) $6 \mathrm{wt} \% \mathrm{MBS}$ ). (A)-(D) and (a)-(d) are same place tested by SEM but with different magnification.

Table 1. The DSC data of PC/PBT alloy with different content of SOG-03.

\begin{tabular}{lccccc}
\hline Samples & $\begin{array}{c}\mathrm{T}_{\mathrm{g}} \text { of PC } \\
\text { phase }\left({ }^{\circ} \mathrm{C}\right)\end{array}$ & $\begin{array}{c}\mathrm{T}_{\mathrm{m}} \text { of PBT } \\
\text { phase }\left({ }^{\circ} \mathrm{C}\right)\end{array}$ & $\begin{array}{c}\Delta \mathrm{H}_{\mathrm{m}} \text { of } \\
\text { PBT phase } \\
(\mathrm{J} / \mathrm{g})\end{array}$ & $\begin{array}{c}\mathrm{T}_{\mathrm{c}} \text { of PBT } \\
\text { phase }\left({ }^{\circ} \mathrm{C}\right)\end{array}$ & $\begin{array}{c}\Delta \mathrm{H}_{\mathrm{c}} \text { of } \\
\text { PBT phase } \\
(\mathrm{J} / \mathrm{g})\end{array}$ \\
\hline PC/PBT-0 & 143.9 & 223.1 & 13.28 & 174.0 & 16.1 \\
PC/PBT-1 & 143.6 & 222.9 & 12.54 & 173.8 & 14.35 \\
PC/PBT-3 & 143.3 & 222.8 & 12.17 & 173.2 & 14.79 \\
PC/PBT-5 & 143.3 & 222.7 & 11.91 & 172.9 & 14.25 \\
PC/PBT-7 & 142.3 & 217.0 & 5.98 & $* *$ & $* *$ \\
\hline
\end{tabular}

Note: ${ }^{*}$ indicates that the PBT phase crystallization peak is weak in the PC/PBT crystallization curve, and no specific data can be obtained. $\mathrm{T}_{\mathrm{g}}$ represents glass transition temperature, $\mathrm{T}_{\mathrm{m}}$ represents fusion temperature, $\Delta \mathrm{H}_{\mathrm{m}}$ represents fusion enthalpy, $\mathrm{T}_{\mathrm{c}}$ represents crystallization temperature, and $\Delta \mathrm{H}_{\mathrm{c}}$ represents crystallization enthalpy.

these two phases. This was because EMA-co-GMA was an ethylene-dominated terpolymer, but the introduction of a large number of methyl acrylate segments that were compatible with PC and PBT changed the polarity of the material. As a result, conventional microscopic analysis was not able to accurately determine its selective dispersion in two phases. In the MBS, the polymethyl methacrylate shell had good compatibility with PC and PBT, and there was no obvious selec- 
tive dispersion in the two phases, so the enlarged section was very smooth.

The enlarged impact profile of the PC/PBT alloys to which SOG-03 was added (Figure 3(c)) showed a distinctly white dispersed phase, which was uniformly dispersed in the matrix resin. It could be found through careful observation that many micropores appeared on the impact section and were mainly dispersed in the white dispersed phase. This was because SOG-03 reacted with PC/PBT alloy in the melt blending process, first acted with PBT with low viscosity and high reactivity, so it was easier to disperse in $\mathrm{PBT}$ phase to achieve toughening thereof. SOG-03 achieved toughening of PBT by functional group reaction. The non-polar POE backbone had no compatibility with PBT, and finally formed a typical morphology of rubber toughened $\mathrm{PBT}$ polymer. During the impact process, the toughened PC phase and the toughened PBT phase were greatly different due to Poisson's ratio. The PBT phase was extruded and even pulled out to form protrusions or sharp corners, when observed by SEM, secondary electrons were easily enriched to form a white dispersed phase structure on the impact section. In order to further verify that SOG-03 tended to be dispersed in the PBT phase in PC/PBT alloy to achieve toughening the alloy, multi-extrusion was conducted to investigate the effect of the order of addition of SOG-03 on the impact property of PC/PBT alloy.

\subsection{The Influence of Multi-Extrusion on the Notched Impact Property of PC/PBT Alloy}

To verify whether SOG- 03 was tended to disperse in PBT phase in PC/PBT phase, multi-extrusion was used to control the reaction sequence of SOG-03 with PC or PBT in the process of preparing the alloy in order to investigate its effect on the impact property. Figure 4 shows the result of multi-extrusion experiment, the notched impact strength of PC/PBT alloy prepared by conventional extrusion process was up to $73.2 \mathrm{KJ} / \mathrm{m}^{2}$. The notched impact strength of $\mathrm{PC} / \mathrm{PBT}$ alloy

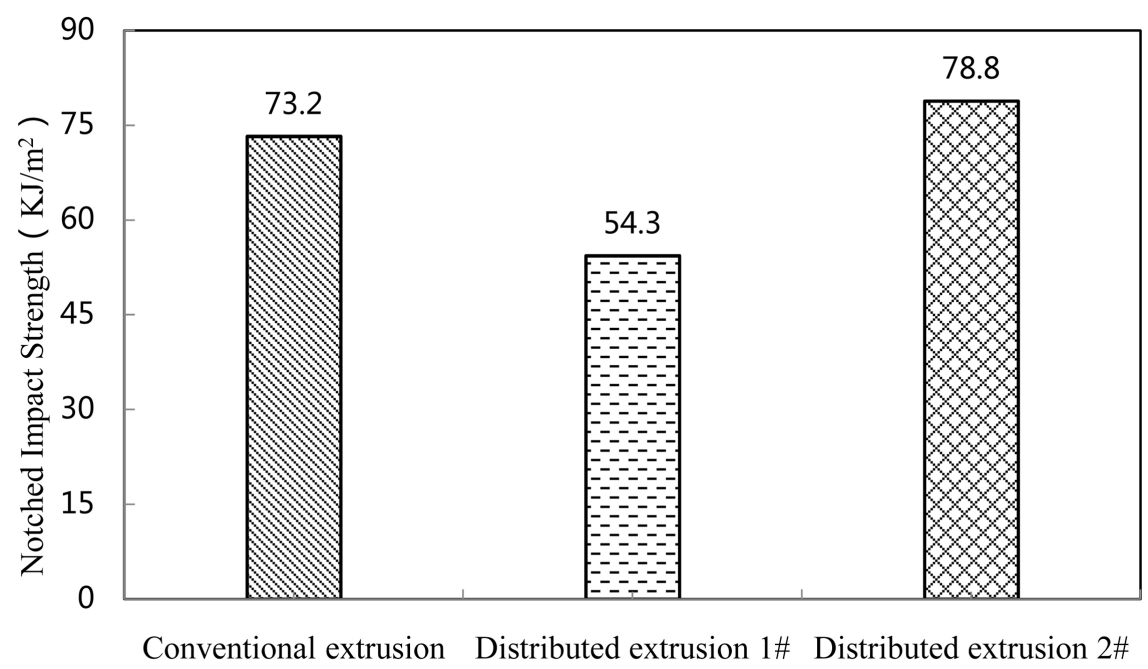

Figure 4. The influence of multi-extrusion on the notched impact strength of PC/PBT alloy. 
prepared by the process that SOG-03 first reacted with PC was only $54.3 \mathrm{KJ} / \mathrm{m}^{2}$, while that could be up to $78.8 \mathrm{KJ} / \mathrm{m}^{2}$ if SOG-03 first reacted with PBT, which was slightly better than $\mathrm{PC} / \mathrm{PBT}$ alloy prepared by conventional extrusion process. SOG-03 first reacted with PBT and dispersed in PBT phase in blending process. SOG-03 dispersed in the PBT phase first achieved toughening of the PBT phase, and finally the formation of the "island structure" $\mathrm{PBT}$ phase was more conducive to the preparation of $\mathrm{PC} / \mathrm{PBT}$ alloy with high impact strength.

\subsection{The Influence of Different Tougheners on Processing Stability of PC/PBT Alloy}

Transesterification in the blending process of $\mathrm{PC} / \mathrm{PBT}$ alloy was a significant factor for deteriorating material properties, and even the addition of transesterification inhibitors did not completely inhibit the occurrence of transesterification during blending. Besides, the transesterification of the alloy material during the later use would still occur slowly, resulting in deterioration of the properties of the alloy. The type of compatible toughener also affected the extent of transesterification in the PC/PBT alloy blending process, in which the degree of stability of the PC/PBT alloy during multi-extrusion (such as appearance, color, melt flow rate, and impact strength) could be indirectly reflected by the addition of different types of toughener.

Photographs of the appearance of a PC/PBT alloy with different types of toughener after different times of multi-extrusion were shown in Figure 5. It could be seen the types of toughener had little effect on the appearance change of

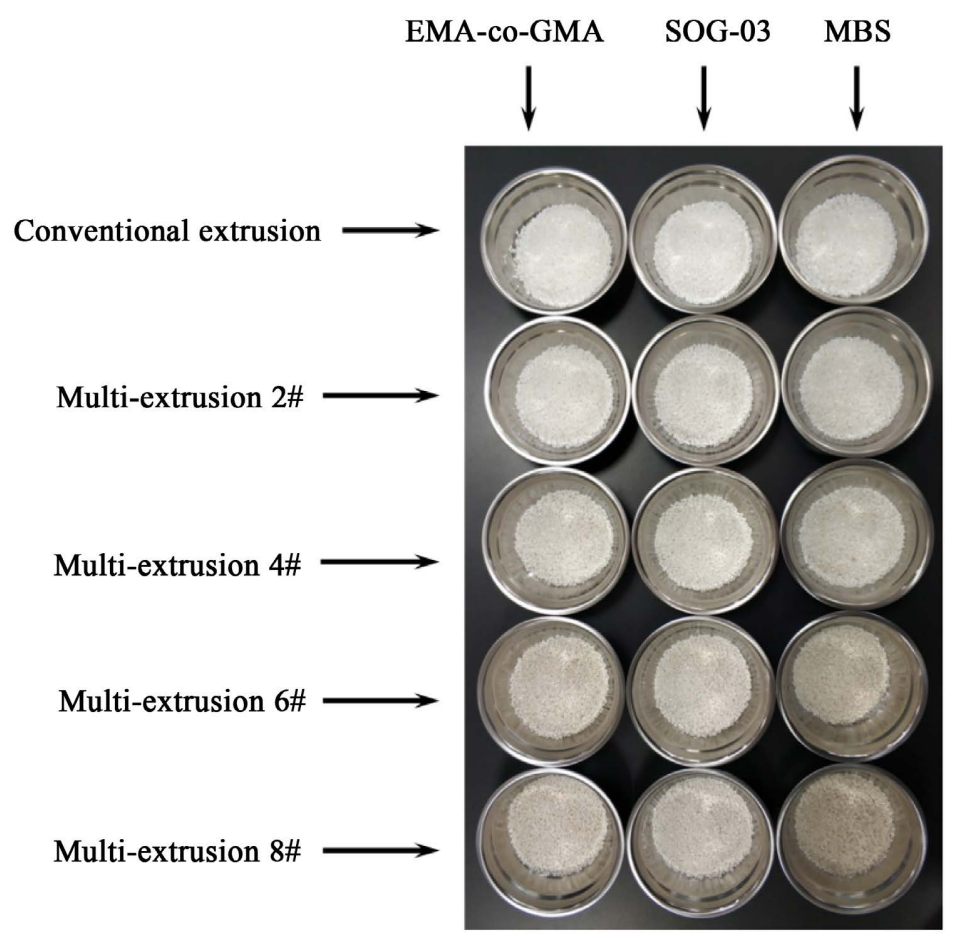

Figure 5. The influence of multi-extrusion process on the appearance of $\mathrm{PC} / \mathrm{PBT}$ alloy with different tougheners. 
PC/PBT alloy during the preliminary stage of conventional and multiple extrusion, this was because the presence of transesterification inhibitors did not allow the alloy material to exhibit the effect of the types of toughener on the appearance of the alloy during conventional extrusion, injection molding, and short-term use. When the times of extrusion were more than 4 , the yellowing of the appearance of the PC/PBT alloy to which MBS was added began to become apparent. With the increase of times of extrusion, the appearance of the PC/PBT alloy to which EMA-co-GMA was added also began to yellow, and the yellowing of the PC/PBT alloy to which SOG-03 was added turned severe. First of all, this was because MBS did not contain functional groups that could react with polyester, and it was impossible to terminate the polyester; then the butadiene rubber phase of the MBS molecule containing a carbon-carbon double bond tended to age and yellow during multi-extrusion process. Although the EMA-co-GMA molecular segment contained a large amount of epoxy functional groups, which could effectively block the PC/PBT alloy at the preliminary stage of multi-extrusion to avoid excessive transesterification, with the increase of the times of extrusion, the EMA main chain with poor heat resistance would decompose the small molecular substance that promoted the transesterification reaction, resulting in the appearance change of the alloy. SOG-03 contained both epoxy functional groups that could cap the alloy, while the highly heat resistant POE backbone ensured a small change in appearance of the alloy during multi-extrusion.

The $\triangle \mathrm{E}$ values of PC/PBT alloy with different types of toughener after different extrusion times were shown in Figure 6. The change trend of $\Delta \mathrm{E}$ values was corresponded with that of appearance shown in Figure 5, the PC/PBT alloy with SOG-03 added had the smallest change in $\Delta \mathrm{E}$ value after multi-extrusion, followed by PC/PBT alloy with EMA-co-GMA, and the $\triangle \mathrm{E}$ value of PC/PBT alloy with MBS changed the most. It was also because the butadiene rubber phase in

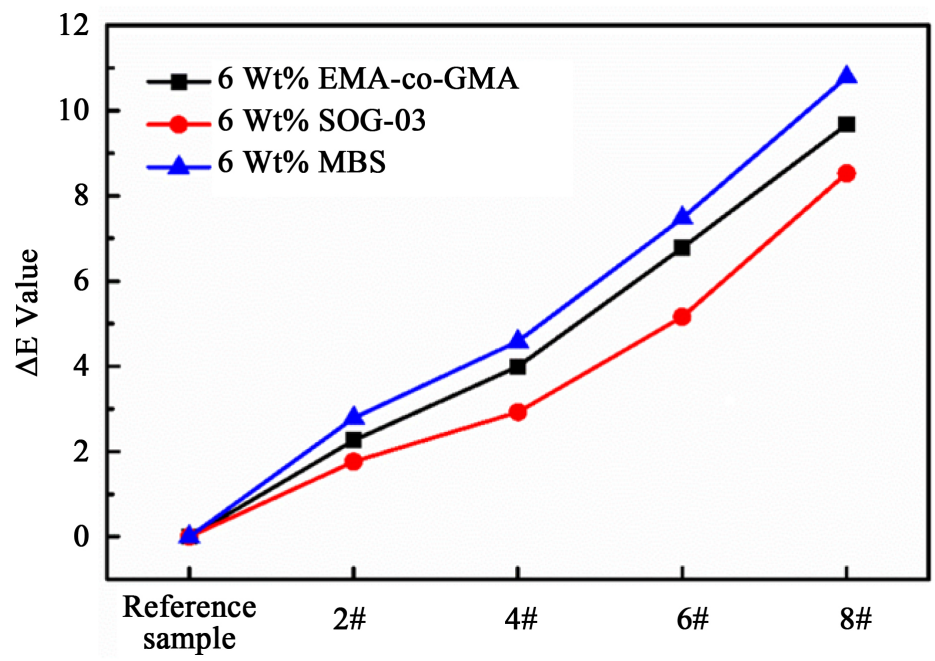

Figure 6. The influence of multi-extrusion process on the $\Delta \mathrm{E}$ value of PC/PBT alloy with different tougheners. 
MBS was easily oxidized and turned yellow during the extrusion process; and the main chain segment of EMA-co-GMA molecule was methyl acrylate, which was less heat-resistant than the main chain of POE, the decomposition of small molecules in multi-extrusion would promote the transesterification reaction, resulting in a higher $\Delta \mathrm{E}$ value than PC/PBT alloy with SOG-03 added.

Melt Flow Rate (MFR) is the main indicator for evaluating the fluidity of materials. The MFR of PC/PBT alloy with different tougheners after multi-extrusion is shown in Figure 7. It could be seen that the MFR of PC/PBT with different tougheners was not significantly different under conventional extrusion; after multi-extrusion, the PC/PBT alloy with SOG-03 added had the smallest increase in MFR, followed by the PC/PBT alloy with EMA-co-GMA, while the MFR of the PC/PBT alloy to which MBS was added was greatly improved, showing poor processing stability. This was because the MBS didn't contain epoxy functional groups and the polyester couldn't be blocked, causing severe transesterification of the PC/PBT alloy during multi-extrusions that resulted in a significant increase in MFR; although the EMA-co-GMA molecular segment had a high epoxy functional group to cap the polyester, the acrylate backbone had poor heat resistance, in the process of multi-extrusion, small molecules would be decomposed to facilitate the transesterification reaction, resulting in that the MFR of PC/PBT alloy with SOG-03 added in the process of multi-extrusion was higher than that of PC/PBT alloy with EMA-co-GMA added.

Similarly, the trend of notched impact strength (Figure 8) of PC/PBT alloy with different tougheners during multi-extrusion process was similar to the trend of appearance, $\triangle \mathrm{E}$ value and MFR. When the same content of toughener was added, the toughening efficiency of MBS was lower than that of PC/PBT alloy with EMA-co-GMA and SOG-03, and the notched impact strength decreased most during the multi-extrusion process. The first was because the butadiene

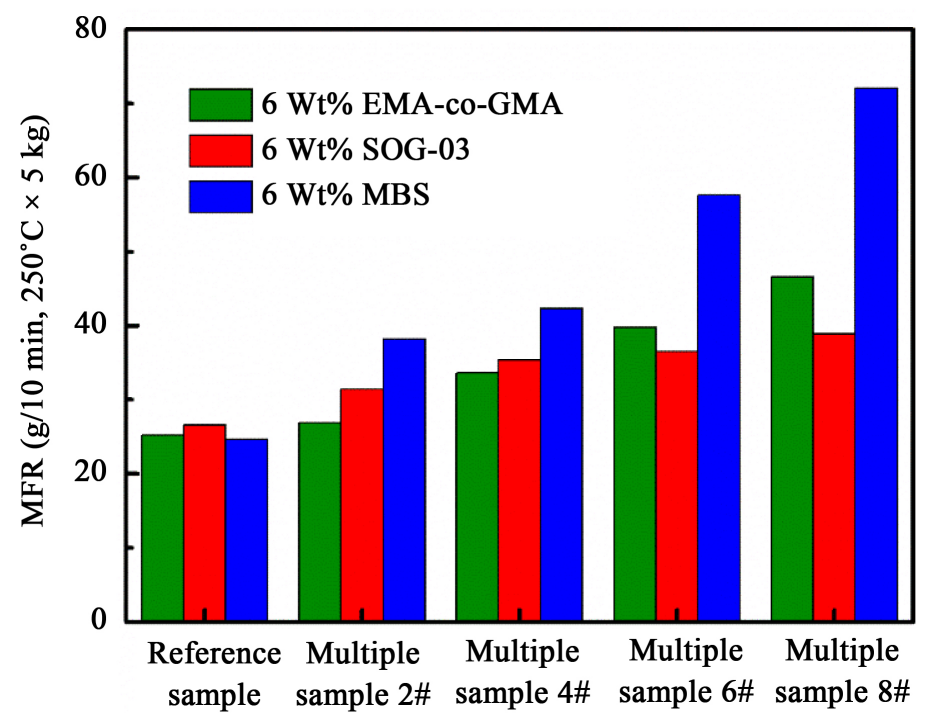

Figure 7. The influence of multi-extrusion process on the MFR of $\mathrm{PC} / \mathrm{PBT}$ alloy with different toughener. 


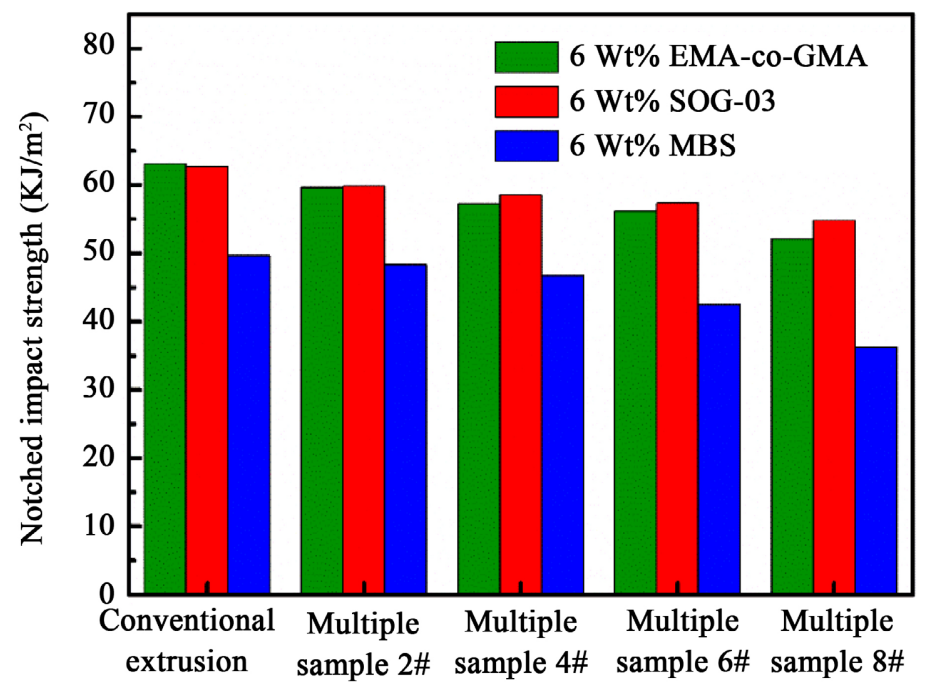

Figure 8. The influence of multi-extrusion process on the notched impact strength of PC/PBT alloy with different tougheners.

phase in MBS was prone to high temperature aging, resulting in a decrease in the impact resistance of the alloy material. Secondly, because the MBS did not contain a reactive functional group, the polyester couldn't be blocked, and the transesterification reaction was intensified during multi-extrusion, resulting in the continuous decrease of notched impact strength of the alloy. The notched impact strength of PC/PBT alloy to which EMA-co-GMA or SOG-03 was added showed a slight downward trend with the increase of multi-extrusion times while that of PC/PBT alloy with SOG-03 added could be maintained better than the PC/PBT alloy with EMA-co-GMA added. This was because although the number of epoxy functional groups in the EMA-co-GMA molecular segment was higher than SOG-03, the heat resistance and toughness of the acrylate backbone were worse than those of the POE chain in SOG-03, during the multi-extrusion process, small molecules were decomposed to promote the transesterification reaction. As a result, after multi-extrusion, the notched impact strength of PC/PBT alloy with EMA-co-GMA added was generally better than that of the PC/PBT alloy to which SOG-03 was added.

Combining the appearance, E value, MFR and notched impact strength shown in Figures 5-8, it can be seen that the addition of saturated polyolefin main chain with high toughness and heat resistance can maintain the processing stability and notched impact strength of PC/PBT alloy to the maximum extent in the multiple extrusion process.

\subsection{The Influence of Long-Term Thermo-0xidative Aging on Impact Property of PC/PBT Alloy}

SOG-03 with high toughness and heat resistance not only maximizes the processing stability and notched impact strength of PC/PBT alloy during multi-extrusion process, but also has excellent long-term thermo-oxidative aging. The influence of long-term thermo-oxidative aging on the impact strength of 


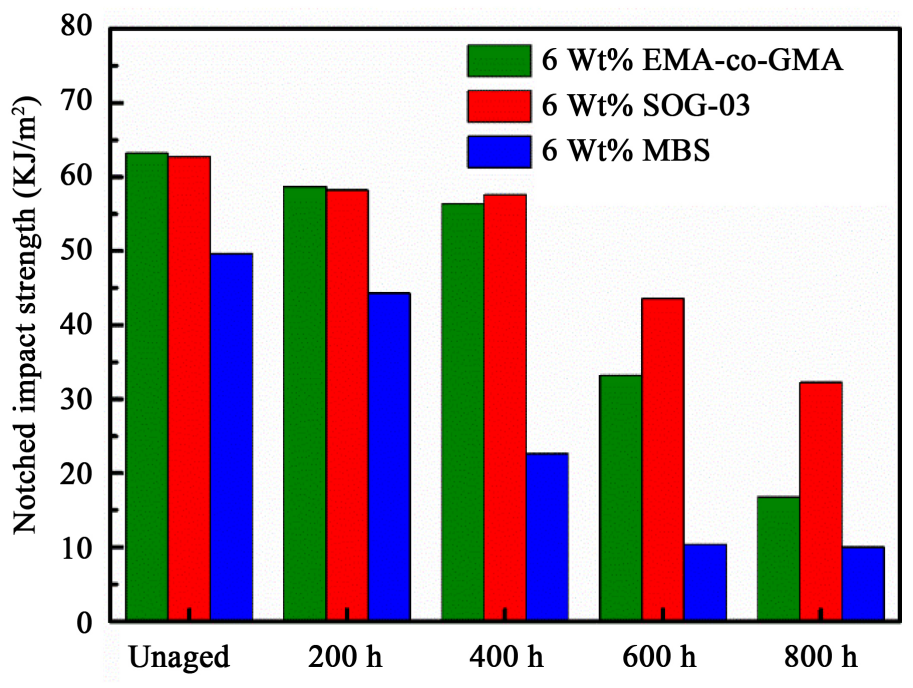

Figure 9. The influence of long-term thermal ageing on the notched impact strength of PC/PBT alloy.

PC/PBT alloy with different tougheners is shown in Figure 9. Similar to the results of multi-extrusion, the MBS toughening efficiency was the lowest when the same content of toughener was added, and the impact strength decreased rapidly during the long-term thermal oxygen aging process. The impact strength of PC/PBT alloys with EMA-co-GMA and SOG-03 added was comparable in the aging time of less than $400 \mathrm{~h}$, and when the aging time was more than $600 \mathrm{~h}$, the impact strength of the alloy with EMA-co-GMA added decreased significantly more than that of the alloy with SOG-03 added. Similarly, because the butadiene phase in MBS was easy to age at high temperature and the unblocked polyester alloy had serious transesterification reaction at high temperature, causing the result that the impact strength of PC/PBT alloy decreases rapidly. In the EMA-co-GMA molecular segment, there was a high epoxy functional group to cap the polyester, and the impact strength did not significantly increase during the short-term thermal aging process. With the prolongation of the hot oxygen aging time, the acrylate backbone with poor heat resistance decomposed into small molecular substance at a high temperature that promoted the transesterification reaction, resulting in a decrease in the impact strength of the PC/PBT alloy. The SOG-03 molecular chain contained both epoxy functional groups that could cap the alloy material, while the high heat-resistant POE backbone ensured that the alloy material maintains high impact strength during long-term thermal oxygen aging.

\section{Conclusion}

In summary, in the $\mathrm{PC} / \mathrm{PBT}$ alloy, brittle-ductile transition began to occur when SOG-03 was added at $3 \mathrm{wt} \%$; SOG-03 tended to disperse in the PBT phase, exhibiting a typical rubber toughened polymer morphology. What's more, compared with EMA-co-GMA and SOG-03, MBS had lower toughening efficiency in 
PC/PBT alloy and more obvious property degradation in multi-extrusion process. PC/PBT alloy with MBS added also had lower impact property than PC/PBT alloy with EMA-co-GMA or SOG-03 added after long-term thermal oxygen aging. Finally, EMA-co-GMA and SOG-03 have the same toughening efficiency in PC/PBT alloys, while the stability and maintaining of impact strength after long-term thermal oxygen aging that the time was more than $600 \mathrm{~h}$ of PC/PBT alloy with SOG-03 added were both better than those of PC/PBT alloy with EMA-co-GMA added.

\section{Conflicts of Interest}

The authors declare no conflicts of interest regarding the publication of this paper.

\section{References}

[1] Jin, Z., Wu, N., et al. (2009) Polycarbonate Resin and Its Application. chemical industry Press, Beijing, 8.

[2] Lu, T., Duan, W., Li, W., et al. (2006) Effect of ktr-6c on the Performance of PC/PBT Blend System. Plastics Industry, 34, 20-22.

[3] Yuan, Z., Peng, H. and Huang, W. (2000) Toughening of PC/PBT Alloy. Aging and Application of Synthetic Materials, 40, 26-31.

[4] Li, C., Qi, Y., Yang, W., et al. (2015) Impact of Four Toughening Agents on PBT Impact Strength and Fluidity. Plastics Industry, 43, 31-35.

[5] Wei, G., Peng, Y., Long, S., et al. (2006) Mechanical Properties and Submicro-Phase States of PBT/PC/E-MA-GMA Ternary Blend System. Synthetic Resin and Plastics, 23, 61-65.

[6] Qian, Z., Yin, J., Li, Z., et al. (2016) Preparation of Chemical-Resistant High-Impact PC/PBT Alloy. Application of Engineering Plastics, 44, 49-51.

[7] Yao, J., Xue, D., Gu, C., et al. (2008) Study on Toughening PC/PBT Alloy with New Toughening Agent. Plastics Industry, 36, 63-65.

[8] Zhu, Y. (2009) Effect of Ethylene-Acrylate-Glycidyl Methacrylate Terpolymer on the Performance of PC/PBT Blend System. Plastics Industry, 36, 31-33. (In Chinese)

[9] Zhu, L., Liu, J. and Zhang, S. (2016) Effect of Toughening Agent MBS on Properties of PC/PBT Alloy. Plastics Industry, 44, 43-47.

[10] Li, C., Shi, P., Tang, J., et al. (2016) Effects of Graft Rate of POE-g-GMA on Properties and Morphology of PBT/POE-g-GMA Blends. Engineering Plastics Applications, 44, 29-34. (In Chinese)

[11] Wang, F., Guo, J., Shao, H., et al. (2011) Preparation of POE Graft GMA and Its Application in Toughening PBT. Plastics Industry, 39, 41-44. (In Chinese)

[12] Li, W., Wang, Y. and Wang, B. (2014) Study on the Effect of PC/PBT Ester Exchange Inhibition on Material Properties. Plastics Manufacturing, 3, 70-80.

[13] Ning, F., Jiang, Z.., Huang, X., et al. (2009) Study on Thermal Aging Behavior of PC/PBT Alloy System. Application of Engineering Plastics, 37, 68-71. 